\title{
Reachability under Contextual Locking
}

\author{
Rohit Chadha ${ }^{1}$, P. Madhusudan ${ }^{2}$, and Mahesh Viswanathan ${ }^{2}$ \\ ${ }^{1}$ LSV, ENS Cachan \& CNRS \& INRIA \\ 2 University of Illinois, Urbana-Champaign
}

\begin{abstract}
The pairwise reachability problem for a multi-threaded program asks, given control locations in two threads, whether they can be simultaneously reached in an execution of the program. The problem is important for static analysis and is used to detect statements that are concurrently enabled. This problem is in general undecidable even when data is abstracted and when the threads (with recursion) synchronize only using a finite set of locks. Popular programming paradigms that limit the lock usage patterns have been identified under which the pairwise reachability problem becomes decidable. In this paper, we consider a new natural programming paradigm, called contextual locking, which ties the lock usage to calling patterns in each thread: we assume that locks are released in the same context that they were acquired and that every lock acquired by a thread in a procedure call is released before the procedure returns. Our main result is that the pairwise reachability problem is polynomial-time decidable for this new programming paradigm as well.
\end{abstract}

\section{Introduction}

In static analysis of sequential programs [7, such as control-flow analysis, dataflow analysis, points-to analysis, etc., the semantics of the program and the data that it manipulates is abstracted, and the analysis concentrates on computing fixed-points over a lattice using the control-flow in the program. For instance, in flow-sensitive context-sensitive points-to analysis, a finite partition of the heap locations is identified, and the analysis keeps track of the set of possibilities of which variables point may point to each heap-location partition, propagating this information using the control-flow graph of the program. In fact, several static analysis questions can be formulated as reachability in a pushdown system that captures the control-flow of the program (where the stack is required to model recursion) 10 .

In concurrent programs, abstracting control-flow is less obvious, due to the various synchronization mechanisms used by threads to communicate and orchestrate their computations. One of the most basic questions is pairwise reachability: given two control locations $p c_{1}$ and $p c_{2}$ in two threads of a concurrent program, are these two locations simultaneously reachable? This problem is very basic to static analysis, as many analysis techniques would, when processing $p c_{1}$, take into account the interference of concurrent statements, and hence would 
like to know if a location like $p c_{2}$ is concurrently reachable. Data-races can also be formulated using pairwise reachability, as it amounts to asking whether a read/write to a location (or an abstract heap region) is concurrently reachable with a write to the same location (or region). More sophisticated verification techniques like deductive verification can also utilize such an analysis. For instance, in an Owicki-Gries style proof [8] of a concurrent program, the invariant at $p c_{1}$ must be proved to be stable with respect to concurrent moves by the environment, and hence knowing whether $p c_{2}$ is concurrently reachable will help determine whether the statement at $p c_{2}$ need be considered for stability.

Pairwise reachability of control locations is hence an important problem. Given that individual threads may employ recursion, this problem can be modeled as reachability of multiple pushdown systems that synchronize using the synchronization constructs in the concurrent program, such as locks, barriers, etc. However, it turns out that even when synchronization is limited to using just locks, pairwise reachability is undecidable [9]. Consequently, recently, many natural restrictions have been identified under which pairwise reachability is decidable.

One restriction that yields a decidable pairwise reachability problem is nested locking [54]: if each thread performs only nested locking (i.e. locks are released strictly in the reverse order in which they are acquired), then pairwise reachability is known to be decidable [5. The motivation for nested locking is that many high-level locking constructs in programming languages naturally impose nested locking. For instance the synchronize(o) $\{\ldots\}$ statement in Java acquires the lock associated with $o$, executes the body, and releases the lock, and hence nested synchronized blocks naturally model nested locking behaviors. The usefulness of the pairwise reachability problem was demonstrated in [5] where the above decision procedure for nested locking was used to find bugs in the Daisy file system. Nested locking has been generalized to the paradigm of bounded lock chaining for which pairwise reachability has also been proved to be decidable $[2,3]$.

In this paper, we study a different restriction on locking, called contextual locking. A program satisfies contextual locking if each thread, in every context, acquires new locks and releases all these locks before returning from the context. Within the context, there is no requirement of how locks are acquired and released; in particular, the program can acquire and release locks in a non-nested fashion or have unbounded lock chains.

The motivation for contextual locking comes from the fact that this is a very natural restriction. First, note that it's very natural for programmers to release locks in the same context they were acquired; this makes the acquire and release occur in the same syntactic code block, which is a very simple way of managing lock acquisitions.

Secondly, contextual locking is very much encouraged by higher-level locking constructs in programming languages. For example, consider the code fragment of a method, in Java [6] shown in Figure 1. The above code takes the lock associated with done followed later by a lock associated with object $r$. In order 


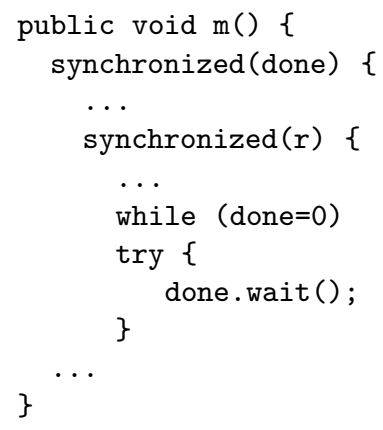

Fig. 1. Synchronized blocks in Java

to proceed, it wants done to be equal to 1 (a signal from a concurrent thread, say, that it has finished some activity), and hence the thread waits on done, which releases the lock for done, allowing other threads to proceed. When some other thread issues a notify, this thread wakes up, reacquires the lock for done, and proceeds.

Notice that despite having synchronized blocks, the wait() statement causes releases of locks in a non-nested fashion (as it exhibits the sequence acq lock_done; acq lock_r; rel lock_done; acq lock_done; rel lock_r; rel lock_done;). However, note that the code above does satisfy contextual locking; the locks $m$ acquires are all released before the exit, because of the synchronized-statements. Thus, we believe that contextual locking is a natural restriction that is adhered to in many programs.

The main result of this paper is that pairwise reachability is decidable under the restriction of contextual locking. It is worth pointing out that this result does not follow from the decidability results for nested locking or bounded lock chains [5]2]. Unlike nested locking and bounded lock chains, contextual locking imposes no restrictions on the locking patterns in the absence of recursive function calls; thus, programs with contextual locking may not adhere to the nested locking or bounded lock chains restrictions. Second, the decidability of nested locking and bounded lock chains relies on a non-trivial observation that the number of context switches needed to reach a pair of states is bounded by a value that is independent of the size of the programs. However, such a result of a bounded number of context switches does not hold for programs with contextual locking. Thus, the proof techniques used to establish decidability are different as well.

We conclude this introduction with a brief outline of the proof ideas behind our decidability result. We observe that if a pair of states is simultaneously reachable by some execution, then they are also simultaneously reachable by what we call a well bracketed computation. A concurrent computation of two threads is not well bracketed, if in the computation one process, say $\mathcal{P}_{0}$, makes a call which is followed by the other process $\left(\mathcal{P}_{1}\right)$ making a call, but then $\mathcal{P}_{0}$ returns from its call before $\mathcal{P}_{1}$ does (but after $\mathcal{P}_{1}$ makes the call). We then observe that every 
well bracketed computation of a pair of recursive programs can simulated by a single recursive program. Thus, decidability in polynomial time follows from observations about reachability in pushdown systems [1].

The rest of the paper is organized as follows. Section 2 introduces the model of concurrent pushdown systems communicating using locks and presents its semantics. Our main decidability result is presented in Section 3. Conclusions are presented in Section 4

\section{Model}

Pushdown Systems. For static analysis, recursive programs are usually modeled as pushdown systems. Since we are interested in modeling threads in concurrent programs we will also need to model locks for communication between threads. Formally,

Definition 1. Given a finite set Lcks, a pushdown system (PDS) $\mathcal{P}$ using Lcks is a tuple $(Q, \Gamma, q s, \delta)$ where

$-Q$ is a finite set of control states.

- $\Gamma$ is a finite stack alphabet.

- qs is the initial state.

$-\delta=\delta_{\text {int }} \cup \delta_{\text {cll }} \cup \delta_{\text {rtn }} \cup \delta_{\text {acq }} \cup \delta_{\text {rel }}$ is a finite set of transitions where

- $\delta_{\text {int }} \subseteq Q \times Q$.

- $\delta_{\mathrm{cll}} \subseteq Q \times(Q \times \Gamma)$.

- $\delta_{\mathrm{rtn}} \subseteq(Q \times \Gamma) \times Q$.

- $\delta_{\text {acq }} \subseteq Q \times(Q \times$ Lcks $)$.

- $\delta_{\text {rel }} \subseteq(Q \times$ Lcks $) \times Q$.

For a $\mathrm{PDS} \mathcal{P}$, the semantics is defined as a transition system. The configuration of a $\mathrm{PDS} \mathcal{P}$ is the product of the set of control states $Q$ and the stack which is modeled as word over the stack alphabet $\Gamma$. For a thread $\mathcal{P}$ using Lcks, we have to keep track of the locks being held by $\mathcal{P}$. Thus the set of configurations of $\mathcal{P}$ using Lcks is Conf $_{\mathcal{P}}=Q \times \Gamma^{*} \times 2^{\text {Lcks }}$ where $2^{\text {Lcks }}$ is the powerset of Lcks.

Furthermore, the transition relation is no longer just a relation between configurations but a binary relation on $2^{\text {Lcks }} \times \operatorname{Conf}_{\mathcal{P}}$ since the thread now executes in an environment, namely, the free locks (i.e., locks not being held by any other thread). Formally,

Definition 2. A PDS $\mathcal{P}=(Q, \Gamma, q s, \delta)$ using Lcks gives a labeled transition relation $\longrightarrow \mathcal{P} \subseteq\left(2^{\text {Lcks }} \times\left(Q \times \Gamma^{*} \times 2^{\text {Lcks }}\right)\right) \times$ Labels $\times\left(2^{\text {Lcks }} \times\left(Q \times \Gamma^{*} \times 2^{\text {Lcks }}\right)\right)$ where Labels $=\{$ int, cll, $\operatorname{rtn}\} \cup\{\operatorname{acq}(l), \operatorname{rel}(l) \mid l \in \mathrm{Lcks}\}$ and $\longrightarrow \mathcal{P}$ is defined as follows.

- fr: $(q, w$, hld $) \stackrel{\text { int }}{\longrightarrow} \mathcal{P}$ fr $:\left(q^{\prime}, w\right.$, hld $)$ if $\left(q, q^{\prime}\right) \in \delta_{\text {int }}$.

- fr : $(q, w$, hld $) \stackrel{\text { cll }}{\longrightarrow} \mathcal{P}$ fr : $\left(q^{\prime}, w a\right.$, hld $)$ if $\left(q,\left(q^{\prime}, a\right)\right) \in \delta_{\text {cll }}$.

- $\mathrm{fr}:(q, w a$, hld $) \stackrel{\text { rtn }}{\longrightarrow} \mathcal{P}$ fr : $\left(q^{\prime}, w\right.$, hld $)$ if $\left((q, a), q^{\prime}\right) \in \delta_{\mathrm{rtn}}$.

- $\mathrm{fr}:(q, w, \mathrm{hld}) \stackrel{\text { acq }(l)}{\longrightarrow} \mathcal{P}$ fr $\backslash\{l\}:\left(q^{\prime}, w\right.$, hld $\left.\cup\{l\}\right)$ if $\left(q,\left(q^{\prime}, l\right)\right) \in \delta_{\text {acq }}$ and $l \in \mathrm{fr}$.

- fr : $(q, w$, hld $) \stackrel{\text { rel }(l)}{\longrightarrow} \mathcal{P}$ fr $\cup\{l\}:\left(q^{\prime}, w\right.$, hld $\left.\backslash\{l\}\right)$ if $\left((q, l), q^{\prime}\right) \in \delta_{\text {rel }}$ and $l \in$ hld. 


\subsection{Multi-pushdown Systems}

Concurrent programs are modeled as multi-pushdown systems. For our paper, we assume that threads in a concurrent program communicate only through locks which leads us to the following definition.

Definition 3. Given a finite set Lcks, a n-pushdown system (n-PDS) $\mathcal{C P}$ communicating via Lcks is a tuple $\left(\mathcal{P}_{1}, \ldots, \mathcal{P}_{n}\right)$ where each $\mathcal{P}_{i}$ is a PDS using Lcks.

Given a $n$-PDS $\mathcal{C P}$, we will assume that the set of control states and the stack symbols of the threads are mutually disjoint.

Definition 4. The semantics of a $n-P D S \mathcal{C P}=\left(\mathcal{P}_{1}, \ldots, \mathcal{P}_{n}\right)$ communicating via Lcks is given as a labeled transition system $T=\left(S, s_{0}, \longrightarrow\right)$ where

$-S$ is said to be the set of configurations of $\mathcal{C P}$ and is the set $\left(Q_{1} \times \Gamma_{1}^{*} \times\right.$ $\left.2^{\text {Lcks }}\right) \times \cdots \times\left(Q_{n} \times \Gamma_{n}^{*} \times 2^{\text {Lcks }}\right)$ where $Q_{i}$ is the set of states of $\mathcal{P}_{i}$ and $\Gamma_{i}$ is the stack alphabet of $\mathcal{P}_{i}$.

$-s_{0}$ is the initial configuration and is $\left(\left(q s_{1}, \epsilon, \emptyset\right), \cdots,\left(q s_{m}, \epsilon, \emptyset\right)\right)$ where $q s_{i}$ is the initial state of $\mathcal{P}_{i}$.

- The set of labels on the transitions is Labels $\times\{1, \ldots, n\}$ where Labels $=$ $\{\mathrm{int}, \mathrm{cll}, \mathrm{rtn}\} \cup\{\operatorname{acq}(l), \operatorname{rel}(l) \mid l \in \mathrm{Lcks}\}$. The labeled transition relation $\stackrel{(\lambda, i)}{\longrightarrow}$ is defined as follows

$$
\left(\left(q_{1}, w_{1}, \text { hld }_{1}\right), \cdots\left(q_{n}, w_{n}, \text { hld }_{n}\right)\right) \stackrel{(\lambda, i)}{\longrightarrow}\left(\left(q_{1}^{\prime}, w_{1}^{\prime}, \text { hld }_{1}^{\prime}\right), \cdots\left(q_{n}^{\prime}, w_{n}^{\prime}, \text { hld }_{n}^{\prime}\right)\right)
$$

iff

Lcks $\backslash \cup_{1 \leq r \leq n} \mathrm{hld}_{r}:\left(q_{i}, w_{i}\right.$, hld $\left._{i}\right) \stackrel{\lambda}{\longrightarrow} \mathcal{P}_{i}$ Lcks $\backslash \cup_{1 \leq r \leq n} \mathrm{hld}_{r}^{\prime}:\left(q_{i}^{\prime}, w_{i}^{\prime}\right.$, hld $\left._{i}^{\prime}\right)$ and for all $j \neq i, q_{j}=q_{j}^{\prime}, w_{j}=w_{j}^{\prime}$ and $\mathrm{hld}_{j}=\mathrm{hld}_{j}^{\prime}$.

Notation: Given a configuration $s=\left(\left(q_{1}, w_{1}, \mathrm{hld}_{1}\right), \cdots,\left(q_{n}, w_{n}, \mathrm{hld}_{n}\right)\right)$ of a $n$-PDS $\mathcal{C P}$, we say that $\operatorname{Conf}_{i}(s)=\left(q_{i}, w_{i}, \operatorname{hld}_{i}\right), \operatorname{CntrlSt}_{i}(s)=q_{i}, \operatorname{Stck}_{i}(s)=$ $w_{i}, \operatorname{LckSt}_{i}(s)=\operatorname{hld}_{i}$ and $\operatorname{StHt}_{i}(s)=\left|w_{i}\right|$, the length of $w_{i}$.

Computations. A computation of the $n$-PDS $\mathcal{C P}$, Comp, is a sequence $s_{0} \stackrel{\left(\lambda_{1}, i_{1}\right)}{\longrightarrow}$ $\ldots \stackrel{\left(\lambda_{m}, i_{m}\right)}{\longrightarrow} s_{m}$ such that $s_{0}$ is the initial configuration of $\mathcal{C P}$. The label of the computation Comp, denoted Label(Comp), is said to be the word $\left(\lambda_{1}, i_{1}\right) \cdots\left(\lambda_{m}, i_{m}\right)$. The transition $s_{j} \stackrel{(\mathrm{cll}, i)}{\longrightarrow} s_{j+1}$ is said to be a procedure call by thread $i$. Similarly, we can define procedure return, internal action, acquisition of lock $l$ and release of lock $l$ by thread $i$. A procedure return $s_{j} \stackrel{(\mathrm{rtn}, i)}{\longrightarrow} s_{j+1}$ is said to match a procedure call $s_{\ell} \stackrel{(\mathrm{cll}, i)}{\longrightarrow} s_{\ell+1}$ iff $\ell<j, \mathrm{StHt}_{i}\left(s_{\ell}\right)=\mathrm{StHt}_{i}\left(s_{j+1}\right)$ and for all $\ell+1 \leq p \leq j$, $\mathrm{StHt}_{i}\left(s_{\ell+1}\right) \leq \operatorname{StHt}_{i}\left(s_{p}\right)$. 
Example 1. Consider the two-threaded program showed in Figure 2, For sake of convenience, we only show the relevant actions of the programs. Figure 3 shows computations whose labels are as follows:

$$
\begin{aligned}
& \begin{array}{l}
\operatorname{Label}(\operatorname{Comp} 1)=(\mathrm{cll}, 0)(\operatorname{acq}(11), 0)(\mathrm{cll}, 1)(\operatorname{acq}(12), 0)(\operatorname{rel}(11), 0)(\operatorname{acq}(11), 1) \\
(\operatorname{rel}(12), 0)(\operatorname{rtn}, 0)(\operatorname{rel}(11), 1)(\operatorname{rtn}, 1)
\end{array} \\
& \text { and } \\
& \begin{array}{r}
\text { Label }(\text { Comp2) }=(\operatorname{cll}, 0)(\operatorname{acq}(11), 0)(\mathrm{cll}, 1)(\operatorname{acq}(12), 0)(\operatorname{rel}(11), 0)(\operatorname{acq}(11), 1) \\
(\operatorname{rel}(11), 1)(\operatorname{rtn}, 1)(\operatorname{rel}(12), 0)(\operatorname{rtn}, 0) .
\end{array}
\end{aligned}
$$

respectively.

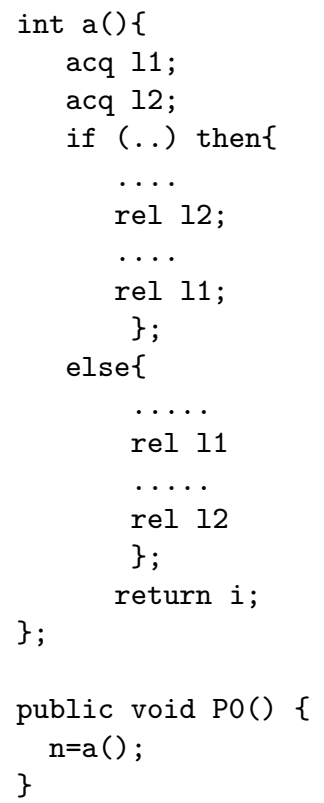

Fig. 2. A 2-threaded programs with threads P0 and P1

\subsection{Contextual Locking}

In this paper, we are considering the pairwise reachability problem when the threads follow the discipline of contextual locking. Informally, this means that -

- every lock acquired by a thread in a procedure call must be released before the corresponding return is executed, and

- the locks held by a thread just before a procedure call is executed are not released during the execution of the procedure. 
$s_{0} \stackrel{\mathrm{cll}}{\longrightarrow} s_{1} \stackrel{\text { acq }(l 1)}{\longrightarrow} s_{2}-\stackrel{\mathrm{cll}}{\longrightarrow} s_{3} \stackrel{\text { acq }(l 2)}{\longrightarrow} s_{4} \stackrel{\text { rel }(l 1)}{\longrightarrow} s_{5} \stackrel{\text { acq }(l 1)}{-} s_{6} \stackrel{\operatorname{rel}(l 2)}{\longrightarrow} s_{7} \stackrel{\text { rtn }}{\longrightarrow} s_{8} \stackrel{\text { rel }(l 1)}{\rightarrow} s_{9}$ rtn $\rightarrow s_{10}$

Comp1

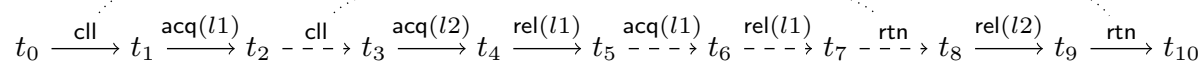

Comp2

Fig. 3. Computations Comp1 and Comp2. Transitions of $P 0$ are shown as solid edges while transition of $P 1$ are shown as dashed edges; hence the process ids are dropped from the label of transitions. Matching calls and returns are shown with dotted edges.

Formally,

Definition 5. A thread $i$ in a $n-P D S \mathcal{C P}=\left(\mathcal{P}_{1}, \ldots, \mathcal{P}_{n}\right)$ is said to follow contextual locking if whenever $s_{\ell} \stackrel{(\mathrm{cll}, i)}{\longrightarrow} s_{\ell+1}$ and $s_{j} \stackrel{(\mathrm{rtn}, i)}{\longrightarrow} s_{j+1}$ are matching procedure call and return along a computation $s_{0} \stackrel{\left(\lambda_{1}, i\right)}{\longrightarrow} s_{1} \ldots \stackrel{\left(\lambda_{m}, i\right)}{\longrightarrow} s_{m}$, we have that

$$
\operatorname{LckSt}_{i}\left(s_{\ell}\right)=\operatorname{LckSt}_{i}\left(s_{j}\right) \text { and for all } \ell \leq r \leq j . \operatorname{LckSt}_{i}\left(s_{\ell}\right) \subseteq \operatorname{LckSt}_{i}\left(s_{r}\right) .
$$

Example 2. Consider the 2-threaded program shown in Figure 2 Both the threads $\mathrm{P} 0$ and $\mathrm{P} 1$ follow contextual locking. The program P2 in Figure 4 does not follow contextual locking.

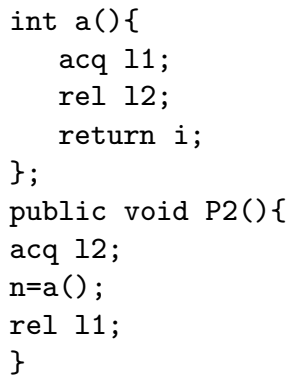

Fig. 4. A program that does not follow contextual locking

Example 3. Consider the 2-threaded program in Figure 5. The two threads P3 and $\mathrm{P} 4$ follow contextual locking as there is no recursion! However, the two 

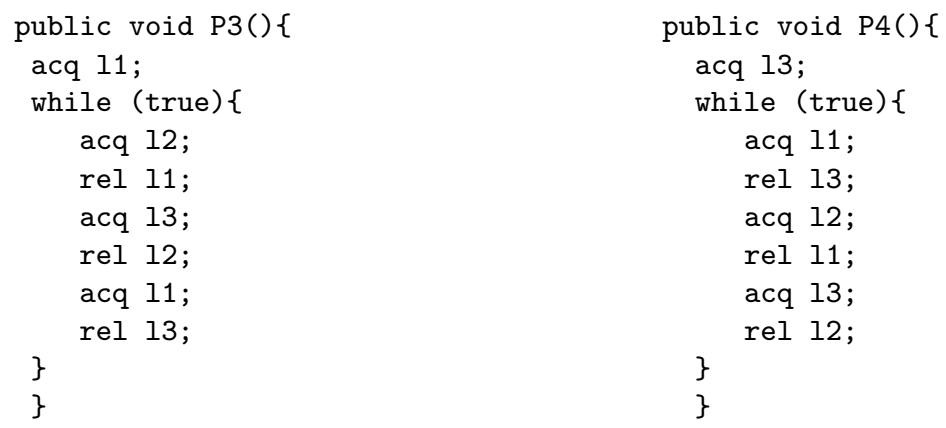

Fig. 5. A 2-threaded program with unbounded lock chains

threads do not follow either the discipline of nested locking [5] or of bounded lock chaining [2]. Hence, algorithms of [5/2] cannot be used to decide the pairwise reachability question for this program. Notice that the computations of this pair of threads require an unbounded number of context switches as the two threads proceed in lock-step fashion. The locking pattern exhibited by these threads can present in any program with contextual locking as long as this pattern is within a single calling context (and not across calling contexts). Such locking patterns when used in a non-contextual fashion form the crux of undecidability proofs for multi-threaded programs synchronizing with locks [5].

\section{Pairwise Reachability}

The pairwise reachability problem for a multi-threaded program asks whether two given states in two threads can be simultaneously reached in an execution of the program. Formally,

Given a $n$-PDS $\mathcal{C P}=\left(\mathcal{P}_{1}, \ldots, \mathcal{P}_{n}\right)$ communicating via Lcks, indices $1 \leq i, j \leq n$ with $i \neq j$, and control states $q_{i}$ and $q_{j}$ of threads $\mathcal{P}_{i}$ and $\mathcal{P}_{j}$ respectively, let $\operatorname{Reach}\left(\mathcal{C P}, q_{i}, q_{j}\right)$ denote the predicate that there is a computation $s_{0} \longrightarrow$ $\cdots \longrightarrow s_{m}$ of $\mathcal{C P}$ such that $\operatorname{CntrlSt}_{i}\left(s_{m}\right)=q_{i}$ and $\operatorname{CntrISt}_{j}\left(s_{m}\right)=q_{j}$. The pairwise control state reachability problem asks if $\operatorname{Reach}\left(\mathcal{C P}, q_{i}, q_{j}\right)$ is true.

The pairwise reachability problem for multi-threaded programs communicating via locks was first studied in [9], where it was shown to be undecidable. Later, Kahlon et. al. 5] showed that when the locking pattern is restricted the pairwise reachability problem is decidable. In this paper, we will show that the problem is decidable for multi-threaded programs in which each thread follows contextual locking. Before we show this result, note that it suffices to consider programs with only two threads [5]. 
Proposition 1. Given a $n-P D S \mathcal{C P}=\left(\mathcal{P}_{1}, \ldots, \mathcal{P}_{n}\right)$ communicating via Lcks, indices $1 \leq i, j \leq n$ with $i \neq j$ and control states $q_{i}$ and $q_{j}$ of $\mathcal{P}_{i}$ and $\mathcal{P}_{j}$ respectively, let $\mathcal{C} \mathcal{P}_{i, j}$ be the $2-P D S\left(\mathcal{P}_{i}, \mathcal{P}_{j}\right)$ communicating via Lcks. Then Reach $\left(\mathcal{C P}, q_{i}, q_{j}\right)$ iff Reach $\left(\mathcal{C P}_{i, j}, q_{i}, q_{j}\right)$.

Thus, for the rest of the section, we will only consider 2-PDS.

\subsection{Well-Bracketed Computations}

The key concept in the proof of decidability is the concept of well-bracketed computations, defined below.

Definition 6. Let $\mathcal{C P}=\left(\mathcal{P}_{0}, \mathcal{P}_{1}\right)$ be a 2-PDS via Lcks and let Comp $=s_{0} \stackrel{\left(\lambda_{1}, i_{1}\right)}{\longrightarrow}$ ... $\stackrel{\left(\lambda_{m}, i_{m}\right)}{\longrightarrow} s_{m}$ be a computation of $\mathcal{C P}$. Comp is said to be non-well-bracketed if there exist $0 \leq \ell_{1}<\ell_{2}<\ell_{3}<m$ and $i \in\{0,1\}$ such that

$-s_{\ell_{1}} \stackrel{(\mathrm{cll}, i)}{\longrightarrow} s_{\ell_{1}+1}$ and $s_{\ell_{3}} \stackrel{(\text { retn }, i)}{\longrightarrow} s_{\ell_{3}+1}$ are matching call and returns of $\mathcal{P}_{i}$, and

- $s_{\ell_{2}} \stackrel{(\mathrm{cll}, i)}{\longrightarrow} s_{\ell_{2}+1}$ is a procedure call of thread $\mathcal{P}_{1-i}$ whose matching return either occurs after $\ell_{3}+1$ or does not occur at all.

Furthermore, the triple $\left(\ell_{1}, \ell_{2}, \ell_{3}\right)$ is said to be a witness of non-well-bracketing of Comp.

Comp is said to be well-bracketed if it is not non-well-bracketed.

Example 4. Recall the 2-threaded program from Example 1 shown in Figure 2. The computation Comp1 (see Figure 3) is non-well-bracketed, while the computation Comp2 (see Figure 3) is well-bracketed. On the other hand, all the computations of the 2-threaded program in Example 3 (see Figure 5) are wellbracketed as the two threads are non-recursive.

The importance of well-bracketing for contextual locking is that if there is a computation that simultaneously reaches control states $p \in \mathcal{P}_{0}$ and $q \in \mathcal{P}_{1}$ then there is a well-bracketed computation that simultaneously reaches $p$ and $q$.

Lemma 1. Let $\mathcal{C P}=\left(\mathcal{P}_{0}, \mathcal{P}_{1}\right)$ be a 2 -PDS communicating via Lcks such that each thread follows contextual locking. Given control states $p \in \mathcal{P}_{0}$ and $q \in \mathcal{P}_{1}$, we have that Reach $(\mathcal{C P}, p, q)$ iff there is a well-bracketed computation $s_{0}^{w b} \longrightarrow$ $\cdots \longrightarrow s_{r}^{w b}$ of $\mathcal{C P}$ such that $\mathrm{CntrlSt}_{0}\left(s_{r}^{w b}\right)=p$ and $\mathrm{CntrlSt}_{1}\left(s_{r}^{w b}\right)=q$.

Proof. Let Comp nwb $=s_{0} \stackrel{\left(\lambda_{1}, i_{1}\right)}{\longrightarrow} \ldots \stackrel{\left(\lambda_{m}, i_{m}\right)}{\longrightarrow} s_{m}$ be a non-well-bracketed computation that simultaneously reaches $p$ and $q$. Let $\ell_{\mathrm{mn}}$ be smallest $\ell_{1}$ such that there is a witness $\left(\ell_{1}, \ell_{2}, \ell_{3}\right)$ of non-well-bracketing of Comp ${ }_{n w b}$. Observe now that it suffices to show that there is another computation Comp mod $_{\text {of }}$ of the same

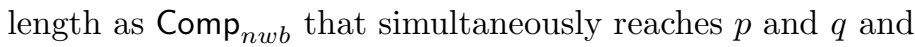


- either Comp mod $_{\text {is }}$ well-bracketed,

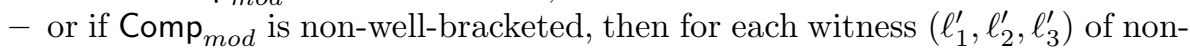
well-bracketing of Comp mod $_{\text {, }}$, it must be the case $\ell_{1}^{\prime}>\ell_{\mathrm{mn}}$.

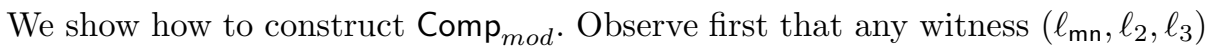

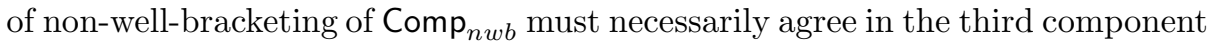
$\ell_{3}$. Let $\ell_{\mathrm{rt}}$ denote this component. Let $\ell_{\mathrm{sm}}$ be the smallest $\ell_{2}$ such that $\left(\ell_{\mathrm{mn}}, \ell_{2}, \ell_{\mathrm{rt}}\right)$ is a witness of non-well-bracketing of Comp mod $_{\text {m }}$. Thus, the transition $s_{\ell_{\mathrm{mn}}} \longrightarrow$ $s_{\ell_{\mathrm{mn}}+1}$ and $s_{\ell_{\mathrm{rt}}} \longrightarrow s_{\ell_{\mathrm{rt}}+1}$ are matching procedure call and return of some thread $\mathcal{P}_{r}$ while the transition $s_{\ell_{s m}} \longrightarrow s_{\ell_{s m}+1}$ is a procedure call by thread $\mathcal{P}_{1-r}$ whose return happens only after $\ell_{\mathrm{rt}}$. Without loss of generality, we can assume that $r=0$.

Let $u,(\mathrm{cll}, 0), v_{1},(\mathrm{cll}, 1), v_{2},(\mathrm{rtn}, 0)$ and $w$ be such that Label $\left(\operatorname{Comp}_{n w b}\right)=$ $u(\mathrm{cll}, 0) v_{1}(\mathrm{cll}, 1) v_{2}(\mathrm{rtn}, 0) w$. and length of $u$ is $\ell_{\mathrm{mn}}+1$, of $u(\mathrm{cll}, 0) v_{1}$ is $\ell_{\mathrm{sm}}+1$. and of $u(\mathrm{cll}, 0) v_{1}(\mathrm{cll}, 1) v_{2}$ is $\ell_{\mathrm{rt}}+1$. Thus, (cll,0) and (rtn,0) are matching call and return of thread $\mathcal{P}_{0}$ and $(\mathrm{cll}, 1)$ is a call of the thread $\mathcal{P}_{1}$ whose return does not happen in $v_{2}$.

We construct Comp mod $_{\text {as follows. Intuitively, Comp }}$ mod is obtained by "rearranging" the sequence Label $\left(\operatorname{Comp}_{n w b}\right)=u(\mathrm{cll}, 0) v_{1}(\mathrm{cll}, 1) v_{2}(\mathrm{rtn}, 0) w$ as follows. Let $v_{2} \mid 0$ and $v_{2} \mid 1$ denote all the "actions" of thread $\mathcal{P}_{0}$ and $\mathcal{P}_{1}$ respectively in $v_{2}$. Then Comp mod $_{\text {mo }}$ is obtained by rearranging $u(\mathrm{cll}, 0) v_{1}(\mathrm{cll}, 1) v_{2}(\mathrm{rtn}, 0) w$ to $u(\mathrm{cll}, 0) v_{1}\left(v_{2} \mid 0\right)(\mathrm{rtn}, 0)(\mathrm{cll}, 1)\left(v_{2} \mid 1\right) w$. This is shown in Figure 6.
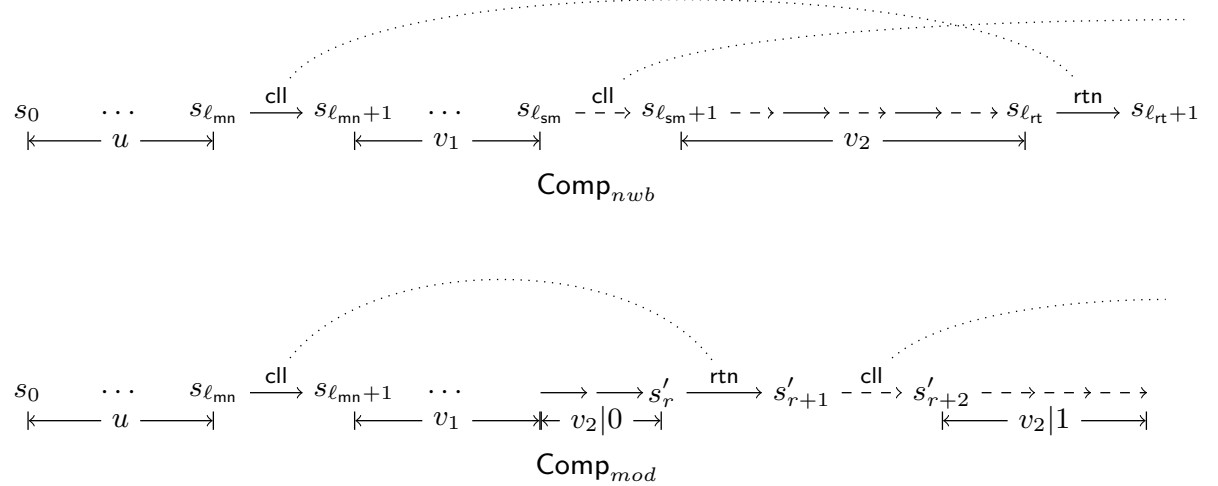

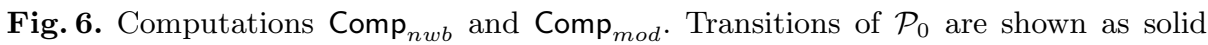
edges and transitions of $\mathcal{P}_{1}$ are shown as dashed edges; hence process ids are dropped from the label of transitions. Matching calls and returns are shown with dotted edges. Observe that all calls of $\mathcal{P}_{1}$ in $v_{1}$ have matching returns within $v_{1}$.

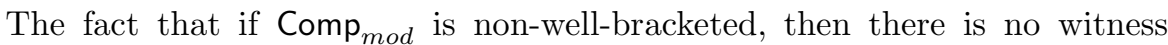
$\left(\ell_{1}^{\prime}, \ell_{2}^{\prime}, \ell_{3}^{\prime}\right)$ of non-well-bracketing with $\ell_{1}^{\prime} \leq \ell_{\mathrm{mn}}$ will follow from the following observations on Label $\left(\operatorname{Comp}_{n w b}\right)$. 
$\dagger v_{1}$ cannot contain any returns of $\mathcal{P}_{1}$ which have a matching call that occurs in $u$ (by construction of $\ell_{\mathrm{mn}}$ ).

t† All calls of $\mathcal{P}_{1}$ in $v_{1}$ must return either in $v_{1}$ or after $c^{\prime}$ is returned. But the latter is not possible (by construction of $\ell_{\mathrm{sm}}$ ). Thus, all calls of $\mathcal{P}_{1}$ in $v_{1}$ must return in $v_{1}$.

Formally, Comp mod $_{\text {is }}$ constructed as follows. We fix some notation. For each $0 \leq$ $j \leq m$, let $\operatorname{Conf}_{0}^{j}=\operatorname{Conf}_{0}\left(s_{j}\right)$ and $\operatorname{Conf}_{1}^{j}=\operatorname{Conf}_{1}\left(s_{j}\right)$. Thus $s_{j}=\left(\operatorname{Conf}_{0}^{j}, \operatorname{Conf}_{1}^{j}\right)$.

1. The first $\ell_{\mathrm{sm}}+1$ transitions of $\mathrm{Comp}_{\text {mod }}$ are the same as Comp $\mathrm{C}_{n w}$, i.e., initially Comp mod $=s_{0} \longrightarrow \cdots \longrightarrow s_{\ell_{\mathrm{sm}}}$.

2. Consider the sequence of transitions $s_{\ell_{\mathrm{sm}}} \stackrel{\left(\lambda_{\mathrm{sm}+1}, i_{\mathrm{sm}+1}\right)}{\longrightarrow} \stackrel{\left(\lambda_{\mathrm{rt}}+1, i_{\mathrm{rt}}+1\right)}{\longrightarrow} s_{\ell_{\mathrm{rt}+1}}$ in Comp. Let $k$ be the number of transitions of $\mathcal{P}_{0}$ in this sequence and let $\ell_{\mathrm{sm}} \leq j_{1}<\cdots<j_{k} \leq \ell_{\mathrm{rt}}$ be the indices such that $s_{j_{n}} \stackrel{\left(\lambda_{j_{n}+1}, 0\right)}{\longrightarrow} s_{j_{n}+1}$. Note that it must be the case that for each $1 \leq n \leq k$

$$
\operatorname{Conf}_{0}^{\ell_{\mathrm{sm}}}=\operatorname{Conf}_{0}^{j_{1}}, \operatorname{Conf}_{0}^{j_{n}+1}=\operatorname{Conf}_{0}^{j_{n+1}} \text { and } \operatorname{Conf}_{0}^{j_{k}+1}=\operatorname{Conf}_{0}^{\mathrm{rt}+1} \text {. }
$$

For $1 \leq n \leq k$, let

$$
s_{\ell_{\mathrm{sm}}+n}^{\prime}=\left(\operatorname{Conf}_{0}^{j_{n}+1}, \operatorname{Conf}_{1}^{\ell_{\mathrm{sm}}}\right) .
$$

Observe now that, thanks to contextual locking, the set of locks held by $\mathcal{P}_{1}$ in Conf $\ell_{1}^{\ell_{\text {sm }}}$ is a subset of the locks held by $\mathcal{P}_{1}$ in Conf $_{1}^{\ell_{j_{n+1}}}$ for each $1 \leq n \leq k$. Thus we can extend Comp mod $_{\text {my }}$ byplying the $k$ transitions of $\mathcal{P}_{0}$ used to

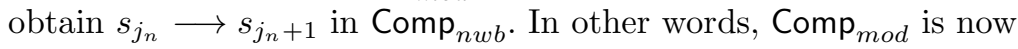

$$
s_{0} \longrightarrow \cdots \longrightarrow s_{\ell_{\mathrm{sm}}} \stackrel{\left(\lambda_{j_{1}+1}, 0\right)}{\longrightarrow} s_{\ell_{\mathrm{sm}}+1}^{\prime} \cdots \stackrel{\left(\lambda_{j_{k}+1}, 0\right)}{\longrightarrow} s_{\ell_{\mathrm{sm}}+k}^{\prime} .
$$

Note that $s_{\ell_{\mathrm{sm}}+k}^{\prime}=\left(\right.$ Conf $_{0}^{\mathrm{rt}+1}$, Conf $\left._{1}^{\ell_{\mathrm{sm}}}\right)$. Thus the set of locks held by $\mathcal{P}_{0}$ in $s_{\ell_{\mathrm{sm}}+k}^{\prime}$ is exactly the set of locks held by $\mathcal{P}_{0}$ at $\operatorname{Conf}_{0}^{\ell_{\mathrm{mn}}}$.

3. Consider the sequence of transitions $s_{\ell_{\mathrm{sm}}} \stackrel{\left(\lambda_{\mathrm{sm}+1}, i_{\mathrm{sm}+1}\right)}{\longrightarrow} \ldots \stackrel{\left(\lambda_{\mathrm{rt}}+1, i_{\mathrm{rt}}+1\right)}{\longrightarrow} s_{\ell_{\mathrm{rt}+1}}$ in Comp. Let $t$ be the number of transitions of $\mathcal{P}_{1}$ in this sequence and let $\ell_{\mathrm{sm}} \leq j_{1}<\cdots<j_{t} \leq \ell_{\mathrm{rt}}$ be the indices such that $s_{j_{n}} \stackrel{\left(\lambda_{j_{n}+1}, 1\right)}{\longrightarrow} s_{j_{n}+1}$. Note that it must be the case that for each $1 \leq n \leq t$,

$$
\operatorname{Conf}_{1}^{j_{1}}=\operatorname{Conf}_{1}^{\ell_{s m}}, \operatorname{Conf}_{1}^{j_{n}+1}=\operatorname{Conf}_{1}^{j_{n+1}} \text { and } \operatorname{Conf}_{1}^{j_{t}+1}=\operatorname{Conf}_{1}^{\mathrm{rt}+1} \text {. }
$$

For $1 \leq n \leq t$, let

$$
s_{\ell_{\mathrm{sm}}+k+n}^{\prime}=\left(\operatorname{Conf}_{0}^{\mathrm{rt}+1}, \mathrm{Conf}_{1}^{j_{n}+1}\right) .
$$

Observe now that, thanks to contextual locking, the set of locks held by $\mathcal{P}_{0}$ in Conf ${ }_{0}^{\ell_{\mathrm{rt}}+1}$ is exactly the set of locks held by $\mathcal{P}_{0}$ at Conf $_{0}^{\ell_{\mathrm{mn}}}$ and the latter is a subset of the locks held by $\mathcal{P}_{0}$ in $\operatorname{Conf}_{1}^{\ell_{n}+1}$ for each $1 \leq n \leq t$. Thus we can extend Comp mod $_{\text {by }}$ applying the $t$ transitions of $\mathcal{P}_{1}$ used to obtain $s_{j_{n}} \longrightarrow s_{j_{n}+1}$ in $\mathrm{Comp}_{n w b}$. In other words, Comp ${ }_{\text {mod }}$ is now

$$
s_{0} \longrightarrow \cdots \longrightarrow s_{\ell_{\mathrm{sm}}+k}^{\prime} \stackrel{\left(\lambda_{j_{1}+1}, 1\right)}{\longrightarrow} s_{\ell_{\mathrm{sm}}+k+1}^{\prime} \cdots \stackrel{\left(\lambda_{j_{t}+1}, 1\right)}{\longrightarrow} s_{\ell_{\mathrm{sm}}+k+t}^{\prime} .
$$




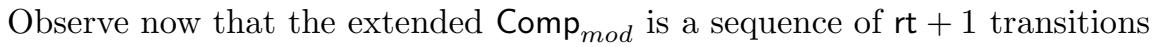
and that the final configuration of Comp ${ }_{m o d}, s_{\ell_{\mathrm{sm}}+k}^{\prime} \stackrel{\left(\lambda_{j_{1}+1}, 1\right)}{=}\left(\operatorname{Conf}_{0}^{\mathrm{rt}+1}, \mathrm{Conf}_{1}^{\mathrm{rt}+1}\right)$ is exactly the configuration $s_{\mathrm{rt}+1}$.

4. Thus, now we can extend Comp mod $_{\text {as }}$

$$
s_{0} \longrightarrow \cdots \longrightarrow s_{\ell_{\mathrm{sm}}+k+t}^{\prime}=s_{\mathrm{rt}+1} \stackrel{\left(\lambda_{\mathrm{rt}+2}, i_{\mathrm{rt}+2}\right)}{\longrightarrow} \cdots \stackrel{\left(\lambda_{m}, i_{m}\right)}{\longrightarrow} s_{m} .
$$

Clearly Comp mod $_{\text {has the same length as Comp }}$ Cwb $_{\text {and simultaneously reaches }}$ $p$ and $q$.

The lemma follows.

\subsection{Algorithm for Deciding the Pairwise Reachability}

We are ready to show that the problem of checking pairwise reachability is decidable.

Theorem 1. There is an algorithm that given a 2-threaded program $\mathcal{C} \mathcal{P}=$ $\left(\mathcal{P}_{0}, \mathcal{P}_{1}\right)$ communicating via Lcks and control states $p$ and $q$ of $\mathcal{P}_{0}$ and $\mathcal{P}_{1}$ respectively decides if Reach $(\mathcal{P}, p, q)$ is true or not. Furthermore, if $m$ and $n$ are the sizes of the programs $\mathcal{P}_{0}$ and $\mathcal{P}_{1}$ and $\ell$ the number of elements of Lcks, then this algorithm has a running time of $2^{O(\ell)} O\left((m n)^{3}\right)$.

Proof. The main idea behind the algorithm is to construct a single PDS $\mathcal{P}_{\text {comb }}=$ $(Q, \Gamma, q s, \delta)$ which simulates all the well-bracketed computations of $\mathcal{C P}$. $\mathcal{P}_{\text {comb }}$ simulates a well-bracketed computation as follows. The set of control states of $\mathcal{P}_{\text {comb }}$ is the product of control states of $\mathcal{P}_{0}$ and $\mathcal{P}_{1}$. The single stack of $\mathcal{P}_{\text {comb }}$ keeps track of the stacks of $\mathcal{P}_{0}$ and $\mathcal{P}_{1}$ : it is the sequence of those calls of the wellbracketed computation which have not been returned. Furthermore, if the stack of $\mathcal{P}_{\text {comb }}$ is $w$ then the stack of $\mathcal{P}_{0}$ is the projection of $w$ onto the stack symbols of $\mathcal{P}_{0}$ and the stack of $\mathcal{P}_{1}$ is the projection of $w$ onto the stack symbols of $\mathcal{P}_{1}$. Thus, the top of the stack is the most recent unreturned call and if it belongs to $\mathcal{P}_{i}$, well-bracketing ensures that no previous unreturned call is returned without returning this call.

Formally, $\mathcal{P}_{\text {comb }}=(Q, \Gamma, q s, \delta)$ is defined as follows. Let $\mathcal{P}_{0}=\left(Q_{0}, \Gamma_{0}, q s_{0}, \delta_{0}\right)$ and $\mathcal{P}_{1}=\left(Q_{1}, \Gamma_{1}, q s_{1}, \delta_{1}\right)$. Without loss of generality, assume that $Q_{0} \cap Q_{1}=\emptyset$ and $\Gamma_{0} \cap \Gamma_{1}=\emptyset$.

- The set of states $Q$ is $\left(Q_{0} \times 2^{\text {Lcks }}\right) \times\left(Q_{1} \times 2^{\text {Lcks }}\right)$.

$-\Gamma=\Gamma_{0} \cup \Gamma_{1}$.

$-q s=\left(\left(q s_{0}, \emptyset\right),\left(q s_{1}, \emptyset\right)\right)$.

$-\delta$ consists of three sets $\delta_{\text {int }}, \delta_{\text {cll }}$ and $\delta_{\text {rtn }}$ which simulate the internal actions, procedure calls, and returns and lock acquisitions and releases of the threads as follows. We explain here only the simulation of actions of $\mathcal{P}_{0}$ (the simulation of actions of $\mathcal{P}_{1}$ is similar). 
- Internal actions. If $\left(q_{0}, q_{0}^{\prime}\right)$ is an internal action of $\mathcal{P}_{0}$, then for each hld $_{0}$, hld $_{1} \in 2^{\text {Lcks }}$ and $q_{1} \in Q_{1}$

$$
\left(\left(\left(q_{0}, \text { hld }_{0}\right),\left(q_{1}, \text { hld }_{1}\right)\right),\left(\left(q_{0}^{\prime}, \text { hld }_{0}\right),\left(q_{1}, \text { hld }_{1}\right)\right)\right) \in \delta_{\text {int }} .
$$

- Lock acquisitions. Lock acquisitions are also modeled by $\delta_{\text {int }}$. If $\left(q_{0},\left(q_{0}^{\prime}, l\right)\right)$ is a lock acquisition action of thread $\mathcal{P}_{0}$, then for each $\mathrm{hld}_{0}$, hld $\mathrm{d}_{1} \in 2^{\text {Lcks }}$ and $q_{1} \in Q_{1}$,

$\left(\left(\left(q_{0}\right.\right.\right.$, hld $\left._{0}\right),\left(q_{1}\right.$, hld $\left.\left._{1}\right)\right),\left(\left(q_{0}^{\prime}\right.\right.$, hld $\left._{0} \cup\{l\}\right),\left(q_{1}\right.$, hld $\left.\left.\left._{1}\right)\right)\right) \in \delta_{\text {int }}$ if $l \notin$ hld $_{0} \cup$ hld $_{1}$.

- Lock releases. Lock releases are also modeled by $\delta_{\text {int }}$. If $\left(\left(q_{0}, l\right), q_{0}^{\prime}\right)$ is a lock release action of thread $\mathcal{P}_{0}$, then for each hld $_{0}, h_{1} d_{1} \in 2^{\text {Lcks }}$ and $q_{1} \in Q_{1}$,

$$
\left(\left(\left(q_{0}, \text { hld }_{0}\right),\left(q_{1}, \text { hld }_{1}\right)\right),\left(\left(q_{0}^{\prime}, \text { hld }_{0} \backslash\{l\}\right),\left(q_{1}, \text { hld }_{1}\right)\right)\right) \in \delta_{\text {int }} \text { if } l \in \text { hld }_{0} .
$$

- Procedure Calls. Procedure calls are modeled by $\delta_{\text {cll }}$. If $\left(q_{0},\left(q_{0}^{\prime}, a\right)\right)$ is a procedure call of thread $\mathcal{P}_{0}$ then hld $_{0}$, hld ${ }_{1} \in 2^{\text {Lcks }}$ and $q_{1} \in Q_{1}$,

$$
\left(\left(\left(q_{0}, \text { hld }_{0}\right),\left(q_{1}, \text { hld }_{1}\right)\right),\left(\left(\left(q_{0}^{\prime}, \text { hld }_{0}\right),\left(q_{1}, \text { hld }_{1}\right)\right), a\right)\right) \in \delta_{\text {cll }}
$$

- Procedure Returns. Procedure returns are modeled by $\delta_{\text {rtn }}$. If $\left(q_{0},\left(q_{0}^{\prime}, a\right)\right)$ is a procedure call of thread $\mathcal{P}_{0}$ then hld $_{0}$, hld h $_{1} \in 2^{\text {Lcks }}$ and $q_{1} \in Q_{1}$,

$$
\left.\left(\left(\left(q_{0}, \text { hld }_{0}\right),\left(q_{1}, \text { hld }_{1}\right)\right), a\right),\left(\left(q_{0}^{\prime}, \text { hld }_{0}\right),\left(q_{1}, \text { hld }_{1}\right)\right)\right) \in \delta_{\text {rtn }} .
$$

It is easy to see that $(p, q)$ is reachable in $\mathcal{C P}$ by a well-bracketed computation iff there is a computation of $\mathcal{P}_{\text {comb }}$ which reaches $\left(\left(p, h_{l} d_{0}\right),\left(q, h_{l} d_{1}\right)\right)$ for some $\mathrm{hld}_{0}, \mathrm{hld}_{1} \in 2^{\text {Lcks }}$. The complexity of the results follows from the observations in [1] and the size of $\mathcal{P}_{\text {comb }}$.

\section{Conclusions}

The paper investigates the problem of pairwise reachability of multi-threaded programs communicating using only locks. We identified a new restriction on locking patterns, called contextual locking, which requires threads to release locks in the same calling context in which they were acquired. Contextual locking appears to be a natural restriction adhered to by many programs in practice. The main result of the paper is that the problem of pairwise reachability is decidable in polynomial time for programs in which the locking scheme is contextual. Therefore, in addition to being a natural restriction to follow, contextual locking may also be more amenable to practical analysis. We observe that these results do not follow from results in [5/4/2 3] as there are programs with contextual locking that do not adhere to the nested locking principle or the bounded lock chaining principle. The proof principles underlying the decidability results are also different. Our results can also be mildly extended to handling programs 
that release locks a bounded stack-depth away from when they were acquired (for example, to handle procedures that call a function that acquires a lock, and calls another to release it before it returns).

There are a few open problems immediately motivated by the results in this paper. First, decidability of model checking with respect to fragments of LTL under the contextual locking restriction remains open. Next, while our paper establishes the decidability of pairwise reachability, it is open if the problem of checking if 3 (or more) threads simultaneously reach given local states is decidable for programs with contextual locking. Finally, from a practical standpoint, one would like to develop analysis algorithms that avoid to construct the crossproduct of the two programs to check pairwise reachability.

For a more complete account for multi-threaded programs, other synchronization primitives such as thread creation and barriers should be taken into account. Combining lock-based approaches such as ours with techniques for other primitives is left to future investigation.

Acknowledgements. P. Madhusudan was supported in part by NSF Career Award 0747041. Mahesh Viswanathan was supported in part by NSF CNS 1016791 and NSF CCF 1016989.

\section{References}

1. Bouajjani, A., Esparza, J., Maler, O.: Reachability Analysis of Pushdown Automata: Application to Model-Checking. In: Mazurkiewicz, A., Winkowski, J. (eds.) CONCUR 1997. LNCS, vol. 1243, pp. 135-150. Springer, Heidelberg (1997)

2. Kahlon, V.: Boundedness vs. unboundedness of lock chains: Characterizing decidability of pairwise CFL-Reachability for threads communicating via locks. In: Proceedings of the IEEE Symposium on Logic in Computer Science, pp. 27-36 (2009)

3. Kahlon, V.: Reasoning about Threads with Bounded Lock Chains. In: Katoen, J.P., König, B. (eds.) CONCUR 2011 - Concurrency Theory. LNCS, vol. 6901, pp. 450-465. Springer, Heidelberg (2011)

4. Kahlon, V., Gupta, A.: An automata-theoretic approach for model checking threads for LTL properties. In: Proceedings of the IEEE Symposium on Logic in Computer Science, pp. 101-110 (2006)

5. Kahlon, V., Ivančić, F., Gupta, A.: Reasoning About Threads Communicating via Locks. In: Etessami, K., Rajamani, S.K. (eds.) CAV 2005. LNCS, vol. 3576, pp. 505-518. Springer, Heidelberg (2005)

6. Lea, D.: Concurrent Programming in Java: Design Principles and Patterns. Addison-Wesley (1999)

7. Muchnick, S.S.: Advanced compiler design and implementation. Morgan Kaufmann Publishers Inc. (1997)

8. Owicki, S.S., Gries, D.: An axiomatic proof technique for parallel programs i. Acta Informatica 6, 319-340 (1976)

9. Ramalingam, G.: Context-sensitive synchronization-sensitive analysis is undecidable. ACM Transactions on Programming Languages and Systems 22(2), 416-430 (2000)

10. Reps, T.W., Horwitz, S., Sagiv, S.: Precise interprocedural dataflow analysis via graph reachability. In: Proceedings of the ACM Symposium on the Principles of Programming Languages, pp. 49-61 (1995) 